\title{
Effect of Cellular Polyanion Mimetics on Tau Peptides Aggregation
}

\author{
Tania Ismail $^{1}$, and Mathumai Kanapathipillai ${ }^{1}$ \\ ${ }^{1}$ University of Michigan-Dearborn, Department of Mechanical Engineering, Dearborn, \\ Michigan, 48128 \\ *E-mail: mathumai@umich.edu
}

\begin{abstract}
Tau protein aggregation is believed to be one of the key drivers of Alzheimer's disease. The two hexapeptide amino acid sequences ${ }^{306} \mathrm{VQIVYK}^{311}$, and ${ }^{275} \mathrm{VQIINK}^{280}$ of the tau protein are responsible for aggregation, and subsequent functional loss leading to Alzheimer's progression. Hence its important to understand the factors that promote the self-aggregation of this tau peptide fragments. Cellular microenvironmental polyanions are known to play a major role in tau protein aggregation and loss of function. Previous studies mainly focused on the effect of heparin and heparan sulfate solution on the full tau protein aggregation. Here we study the effect of cellular polyanion mimetics, glycosaminoglycans (GAGs) in solution and on surface to mimic intracellular and extracellular conditions, and cellular RNA on the aggregation of tau hexapeptide ${ }^{306}$ VQIVYK ${ }^{311}$, and mutant tau peptide ${ }^{273}$ GKVQIIN_KLDL ${ }^{284}$ found frequently in taupathy. Characterization techniques used for the study include, biochemical assays, electron microscopy, and spectroscopy techniques to investigate the aggregation vitro.
\end{abstract}

This is the author manuscript accepted for publication and has undergone full peer review but has not been through the copyediting, typesetting, pagination and proofreading process, which may lead to differences between this version and the Version of Record. Please cite this article as doi: $10.1002 /$ psc.3125

This article is protected by copyright. All rights reserved. 
Results show while all the GAGs in solution form play a role in tau peptides aggregation, heparin, chondroitin sulfate, and dermatan sulfate in particular significantly promote the tau peptides aggregation formation. On the other hand surface coated GAGs did not promote significant tau aggregation. As far as cellular RNA is concerned, results show significant increase in tau peptides aggregation and toxicity in neuroblastoma cells. The study on the tau peptides fragments aggregation in the presence of GAGs and cellular RNA, could provide novel information on the tau aggregation mechanism, and could open ways for developing cellular polyanions modulating therapeutics for treating Alzheimer's disease.

Key words: glycosaminoglycans, RNA, aggregation, tau peptide, alzheimer's

\section{Introduction}

Tau protein aggregation and subsequent neurofibrillary tangle formation are major hallmarks of Alzheimer's disease. Recent reports suggest understanding the tau aggregation mechanism could help develop clinically relevant therapies in combating the Alzheimer's disease [1, 2]. Tau protein consists of 441 amino acids with microtubule binding region that contains four repeat peptide units [3, 4]. They are composed of 
hexapeptide domains, which are believed to be the major drivers of tau pathological assembly. The hexapeptide repeats ${ }^{306} \mathrm{VQIVYK}^{311}$, and ${ }^{275} \mathrm{VQIINK}^{280}$, of the tau protein have been shown to form inter-tau beta sheets, aggregates, and fibril structures [5-9]. The hexapeptide repeats are well studied, and hence is widely used as a model to study tau aggregation in vitro.

Several factors are known to influence the tau aggregation [5, 10-12]. Among them, polyanions, glycosaminoglycans (GAGs) and their sulfated forms, and cellular RNA have shown to induce Alzheimer's like changes in tau protein and believed to play a major role in tau aggregation $[13,14]$. Further, sulfated glycosaminoglycans have shown to promote tau aggregation and have been found to be present in Alzheimer's disease affected brain $[13,15]$. Hence it is important to understand the influence of the polyanions on tau aggregation. Previously, it has been shown that GAGs influence the aggregation of amyloid beta peptide $\left(\mathrm{A} \beta_{42}\right)$ aggregation in vitro [16]. Also it has been reported that tau assemble into filaments like morphology in the presence of sulfated heparin [17], and heparin induced conformational changes in tau protein have been observed $[18,19]$. Further, recently NMR studies on tau-glycans interactions have been reported, where it has been shown that 6-O-sulfation in heparin is important for tau interaction [20]. In another study, tau peptide fragments aggregation in the presence of heparin has been shown to shift the oligomeric conformation of tau and contribute to the subsequent fibril 
formation kinetics and morphology of the final aggregates [21]. However, the effect of other glycosaminoglycan molecules on tau hexapeptides aggregation under intracellular and extracellular mimetic conditions hasn't been explored in detail. In the extracellular conditions GAGS are displayed on the extracellular matrix surface, while in the intracellular conditions, they are present in the solution. Given that tau aggregation has been observed both in intra-cellular and extracellular microenvironment [22, 23], it is important to test the tau aggregation in the presence of GAGs both in solution and on surface. Further, effect of cellular RNA on the tau peptides aggregation is yet to be explored as well.

Here in this paper we study the effect of GAG molecules heparin (Hep), heparan sulfate (HepS), dermatan sulfate (DS), chondroitin sulfate (CS), and hyaluronic acid (HA) in solution and on surface, and cellular RNA, on the aggregation of tau hexapeptide domain ${ }^{306}$ VQIVYK $^{311}$ (Tau V) segment from the microtubule binding region of tau protein, and the $\Delta$ K280 mutant peptide fragment ${ }^{273}$ GKVQIIN_KLDL ${ }^{284}($ Tau $N)$ widely found in taupathy. The aggregation of the peptides in the presence of polyanions was studied utilizing the biochemical, microscopy, and spectroscopy characterization methods, thioflavin S fluorescence assay, transmission electron microscopy (TEM), atomic force microscopy (AFM), turbidity, dynamic light scattering (DLS), and cell toxicity assay. The results show that the heparin, chondroitin sulfate, and dermatan sulfate in solution, 
significantly promote tau peptides aggregation and fibril formation, while surface coated GAGs did not promote significant aggregation. Further, cellular RNA significantly promoted the peptides aggregation as well. The study demonstrates for the first time the effect of cellular polyanion mimetics on tau peptides aggregation, and hence could provide added information on the role of polyanions in tau aggregation.

\section{Materials and Methods}

\section{Materials}

Tau peptides (Ac-VQIVYK-NH ${ }_{2}$, Ac-GKVQIINKLDL-NH $\mathrm{N}_{2}$ were custom synthesized from Genscript. Low molecular weight heparin, heparan sulfate (GAG-HS01), dermatan sulfate (GAG-DS01), and hylaluronic acid (HA93), were purchased from Galen Laboratory Supplies. Chondroitin sulfate (C4384), all other chemicals, and reagents were purchased from Sigma Aldrich. Heparin/GAG binding plates (SKU : H/G plates) was purchased from Galen Laboratory Supplies, and the PureLink RNA mini kit was purchased from Thermo Fisher Scientific.

\section{ThS fluorescence measurements}

First stock solutions of tau peptides $(1 \mathrm{mM})$ and GAGs $(1 \mathrm{mM})$ were prepared by dissolving in deionized water. ThS was freshly prepared at a concentration of $0.5 \mathrm{mg} / \mathrm{ml}$ 
in $20 \mathrm{mM}$ MOPS buffer, $\mathrm{pH}$ 7. For the ThS measurement, $10 \mu \mathrm{l}$ of peptide was dissolved in $2.5 \mu \mathrm{l} \mathrm{GAG} \mathrm{stock} \mathrm{solution,} 10 \mu \mathrm{l} \mathrm{ThS}$, and MOPS buffer to make the final $100 \mu \mathrm{l}$ working solution. In the case of RNA, $0.25 \mathrm{ng} / \mathrm{ml}$, and $0.5 \mathrm{ng} / \mathrm{ml}$ of final RNA concentrations was used with $100 \mu \mathrm{M}$ of final tau peptide concentrations. A M3 spectramax spectrophotometer was used to measure the ThS fluorescence at 440/490 nm, excitation and emission. Aggregation kinetic measurements were performed every two minutes for 30 minutes, in 96 black well plates. At least three independent experiments were carried out and the average readings were obtained.

\section{DLS}

A Malvern zetasizer instrument in the lab was used to measure the size of the aggregation of the peptides with GAGs. Samples were prepared under similar conditions used for the ThS measurements, but without the ThS, and the size measurements were performed. Three independent size measurement experiments were performed for each GAGs conditions.

\section{Turbidity measurements}

The peptides aggregation was measured by the turbidity of the samples at $400 \mathrm{~nm}$. Samples were prepared under similar conditions used for the ThS study, but without the $\mathrm{ThS}$, and the turbidity measurements were performed using a spectramax M3 
spectrophotometer in the lab. Three independent size measurement experiments were performed for each GAGs conditions.

\section{TEM}

TEM images were obtained using the JEOL JSM 1400 TEM at UM-Ann arbor electron microscope facility. The peptides were aggregated with GAGs for 1 hour, spotted on holy carbon copper grids, and then stained with $1 \%$ uranyl formate.

\section{Cell toxicity assay}

The toxicity of tau peptides aggregates with GAGs on human neuroblastoma SH-SY5Y cells was studied by the XTT assay. Cells were obtained from American Type Culture Collection (ATCC), and cultured according to the protocol. For the study, $2 \times 10^{4}$ cells/well, were cultured in 96 well plates over night. Peptides $(32 \mu \mathrm{M})$ with or without GAGs of concentrations $(8 \mu \mathrm{M})$ were aggregated for 24 hours, and subsequently incubated for 48 hours. For RNA induced toxicity, peptides $(16 \mu \mathrm{M})$ with or without RNA of $0.8 \mathrm{ng} / \mathrm{ml}$, was used. XTT assay was measured at $470 \mathrm{~nm}$, and the viability was determined according to manufacturer's protocol.

This article is protected by copyright. All rights reserved. 


\section{GAGs surface coating and aggregation assays}

To study the influence of surface coated GAGs on peptide aggregation, first GAGS were coated on GAGs binding plates. For GAGs binding, $100 \mu$ l solution of $0.01 \mathrm{M}$ PBS containing $25 \mu \mathrm{M}$ of GAGs (Hep, CS, DS, HepS, and HA) were added to a GAGs binding plate (Heparin/GAG binding plates (SKU : H/G plates), and incubated at room temperature according to manufacturer's protocol. After 24 hours, the PBS/GAGs solution was aspirated and the wells were washed three times with PBS buffer. For aggregation studies on GAGs coated plates, $15 \mu \mathrm{l}$ of $1 \mathrm{mM}$ Tau N, $15 \mu \mathrm{ThS}(0.5 \mathrm{mg} / \mathrm{ml})$, and $120 \mu \mathrm{l}$ MOPS $(20 \mathrm{mM})$ solution was added into each well. The turbidity of the peptide aggregates was measured at $400 \mathrm{~nm}$ wavelength after $30 \mathrm{~min}$ incubation. The morphology of the aggregation was characterized by AFM.

\section{RNA Extraction}

Human brain microvascular endothelial cells (hBMVEC) were cultured at a density of $\leq$ $5 \times 10^{6}$ cells/ml. PureLink RNA mini kit was used to extract the RNA from the cells, according to the manufacturer's protocol. Using the Spectra-Drop protocol in spectramax M3 spectrophotometer, the absorbance was measured from $230 \mathrm{~nm}-280 \mathrm{~nm}$. Then, using the measured optical density (OD) at $260 \mathrm{~nm}$ and the provided standard curve (OD 260 nm vs. concentration), the concentration of RNA was obtained. The purified RNA was stored at $-80^{\circ} \mathrm{C}$ until used for the aggregation studies. 


\begin{abstract}
AFM
Hitachi AFM 5100N was used to obtain the AFM images for tau peptides samples with GAGs and RNA. For preparation, $2 \mu \mathrm{l}$ of the aggregated sample solutions were spotted on freshly cleaved mica, and kept to dry, and washed it with water to remove salt, and dried again before imaging. AFM tips (Applied Nanostructures Inc.) with an average resonance frequency of $300 \mathrm{kHz}$, was used to image samples using tapping mode. The length and the width of the cantilever are $125 \mu \mathrm{m}$ and $30 \mu \mathrm{m}$ respectively, and the average force constant is $37 \mathrm{~N} / \mathrm{m}$.
\end{abstract}

\title{
Statistical Analysis
}

Each experiments were conducted three or more times, and they are presented as mean \pm standard error of the mean (SEM). P-values were determined from the results of at least 3-independent experiments. For statistical significance unpaired t-test was used. Each GAGs conditions were compared to the controls without GAGs. ${ }^{* *} p<0.01,{ }^{*} p<0.05$ are considered as significance.

\section{Results and Discussion}

The goal of the study is to test the influence of polyanions on tau peptides aggregation, subsequent fibril formation, and cellular toxicity. For the GAGS induced aggregation 
study, peptides to GAGs molar ratios of $4: 1$ or $20: 1$ were used, at a constant tau peptide concentrations of $100 \mu \mathrm{M}$. The starting molar ratio 4:1 and tau concentration $(100 \mu \mathrm{M})$ were chosen as previously reported [24,25], while the 20:1 molar ratio was tested to see if the tau peptide: GAGs concentration ratio has an effect on the aggregation. Thioflavin $\mathrm{S}$ measurements show that the GAGs influence both Tau V, and Tau $\mathrm{N}$ peptides aggregation in vitro. The enhanced ThS signal in the presence of GAGs may be due to the presence of aggregates amount or due to the differences in aggregates structure. Increased thioflavin signal of the peptides was observed in the presence of chondroitin sulfate, heparin, and dermatan sulfate (Figure 1A, B). Heparan sulfate and hylaluronan on the other hand exhibited minimal thioflavin S fluorescence. At both 4:1, and 20:1 molar ratios, chondroitin sulfate seems to exhibit enhanced thioflavin $\mathrm{S}$ binding of Tau $\mathrm{V}$ peptide (Figure 1A, Figure S1A). Tau N ThS binding is enhanced in the presence of chondroitin sulfate at lower GAG molar ratios (20:1) (Figure S1B), while at higher GAG molar ratios $(4: 1)$, heparin seems to have more influence on the thioflavin $S$ binding (Figure 1B). This may be due to the influence of heparin concentration on tau interaction whereas chondroitin sulfate does not seems to influence aggregation at higher molar ratio. Also it is important to note that, heparin exhibited significant aggregation (p-value $<0.01)$ compared to the sulfated form of heparin revealed by t-test for the measurements (heparin vs heparin sulfate) performed for the ThS the kinetics. 
DLS, and turbidity measurements were then used to measure the size distribution, and turbidity of the peptide aggregates, with and without GAGs. As can be seen from Figure 2A, chondroitin sulfate, heparin, and dermatan sulfate treated Tau N peptides, exhibited larger sizes, indicating the influence on aggregation or fibrillar structures, however significance increase in size was observed with chondroitin sulfate treated samples only. Similarly, as for the Tau V peptide aggregation size, although increase in the size for all the GAGs treated peptides was observed compared to Tau V peptide alone, chondroitin sulfate treated peptide samples exhibited significant increase in aggregation size (Figure 2B). It is also important to point out that the DLS measurements may be influenced by the conformational heterogeneity and the size of the aggregates, and hence is an approximate method to predict the aggregates size. The turbidity measurements reveal significant turbidity with chondroitin sulfate, heparin, heparan sulfate, and dermatan sulfate treated peptides compared to Tau V and Tau $\mathrm{N}$ peptides alone (Figure 3). Hyaluronic acid on the other hand, exhibited no significant turbidity with both peptides. However, DLS measurements revealed that TauV samples treated with hyaluronic acid showed larger size aggregates (Figure 2B), this may be due to the formation of few globular aggregates, which may have contributed to larger size, but not significant turbidity. 
Next we tested the morphology of the Tau $\mathrm{N}$ and TauV aggregates by TEM (Figure 4, 5). Images reveal, that the Tau $\mathrm{N}$ peptides treated with heparin, and chondroitin sulfate exhibited significant amount of fibril like structures (Figure 4), in agreement with the ThS measurements. Dermatan sulfate treated peptides exhibited aggregates like morphology. Also in agreement with the ThS measurements, the TEM images of heparan sulfate, and hyaluronic acid treated samples showed no significant aggregation compared to other GAGs treated samples. Similar observations were found with Tau V aggregated samples (Figure 5), although the amount of aggregation/fibrillar morphology seems to be less compared to Tau $\mathrm{N}$ aggregates. This is in agreement with $\mathrm{ThS}$ measurement, where higher fluorescence intensities were observed with Tau $\mathrm{N}$ samples compared to Tau V samples.

Next we tested whether aggregation or fibrillar morphologies were observed under extracellular mimetic conditions of GAGSs. For this, GAGs were coated on special GAGs binding plates, and then Tau $\mathrm{N}$ peptides solutions were subsequently incubated on the surface to allow aggregation. As can be seen from AFM images in Figure S2 A, all the GAGs exposed peptides showed aggregates like morphology. However, the amount of aggregates seems to be not significant among the GAGS treated and non-treated samples. To further confirm this, we did turbidity measurements. TauN peptide buffer solutions were incubated on GAGs coated plates and allowed to aggregate. After 30 
minutes, the supernatant of the samples were taken and turbidity measurements were performed. As can be seen from the turbidity study shown in Figure S2 B, there is no significant difference among different GAGS treatment compared to untreated GAGs. However, among the GAGs, chondroitin sulfate treated samples exhibited more aggregation/fibril formation, revealed from both AFM and turbidity. Overall, the data suggests, that the intracellular GAGs may have major role in tau aggregation compared to extracellular GAGs.

To assess the toxicity effects of GAGs on tau peptide aggregation, we performed cellular toxicity studies of Tau $\mathrm{N}$ and Tau V aggregated samples in the presence of GAGs. For the study, we used SH-SY5Y human neuroblastoma cells, that is widely used as a model cell line in neurodegenerative disease studies to assess the toxicity effects [26]. Cells were cultured in 96 well plates and incubated with Tau peptides and GAGs solution for 48 hours and XTT assay was performed to assess the cell viability. As can be seen from the Figure 6, all the samples treated with GAGs in solution exhibited toxicity effects. While dermatan sulfate treated samples showed a slight increase in toxicity, statistically it is not significantly different from other GAGs treated samples. The enhanced toxicity in the presence of dermatan sulfate may be due to its influence on the formation of tau peptides aggregates like morphology, compared to fibrillar structures as observed from the TEM and AFM images. In addition, the peptides alone also show some toxicity 
effects, may be due to the influence of extracellular GAGs, deposited by the cells. To confirm the toxicity effects are due to the peptides aggregation and not due to the GAGs itself, we performed the toxicity studies with GAGs only. GAGs alone in solution exhibited no significant toxicity (Figure S3). Polyanions including heparin have shown to promote tau and tau peptide aggregation by binding to the microtubule hexapeptide regions and with the positively charged lysine and histidine aminoacid chains [12]. GAGs, which are polyanionic, seem to promote tau aggregation in a similar manner, and subsequently exhibit toxic effects on the cells.

As a next step, we tested the role of cellular RNA on Tau N and Tau V peptides aggregation. For the study we used 0.25 , and $0.5 \mathrm{ng} / \mathrm{ml}$ of cellular RNA with $100 \mu \mathrm{M}$ of peptide final concentrations. Both peptides showed enhanced aggregation in the presence of RNA, observed from increase in ThS fluorescence (Figure 7A, B). To determine whether it has similar aggregation kinetics like GAGs, we calculated the rate constants of TauN and Tau V in the presence of chondroitin sulfate, heparin, and RNA. The ThS curves were fitted using four-parameter sigmoidal curve using Matlab with the following equation $y=y o+\frac{a}{1+e^{-(x-x o) / b}}$, here $y o-$ initial $\mathrm{ThS}$ fluorescence, $x o-50 \%$ of the maximum fluorescence value, a-maximal fluorescence value, $y$ and $x$ are the ThS fluorescence, and time, as previously reported in literature to determine protein 
aggregation kinetics [27]. As can be seen from Table 1, the RNA and GAGs influence on the aggregation kinetics is comparable within the peptides. However, Tau V in the presence of GAGs and RNA showed faster kinetics compared to Tau N samples. This is further evidenced by AFM imaging where Tau V treated with RNA exhibited more aggregates compared to Tau $\mathrm{N}$ in general (Figure 7C). From the images, Tau $\mathrm{N}$ seems to drive more fibril like structures, while Tau V forms more globular aggregates. Finally, the RNA mediated Tau V and Tau N aggregates toxicity was assessed in SH-SY5Y cells. Tau V exhibited significant toxicity, whereas Tau $\mathrm{N}$ did not exhibit toxicity at the tested concentrations (Figure 8). This may be due to the significant Tau V aggregation formation in the presence of RNA (Figure 7).

Glycosaminoglycans have shown to play a key role in Alzheimer's disease. A recent study reveal that increase evidence suggest that $\mathrm{N}$-glycans may play an important role in Alzheimer's disease [28]. In another study, it is stated that GAGs have a scaffolding role on amyloidogenic proteins [29]. Further, it has been reported that glycosaminoglycan heparin, which is anionic in nature, believed to play a key role in the tau aggregation by interacting with the microtubule binding region $[13,18]$. RNA also known to play a key role in tau aggregation. Kampers et. al has shown that in the presence bovine or yeast RNA, the tau was assembled into paired helical filaments [14]. Here we show that GAGS, and human brain endothelial cellular RNA in solution have the capability to 
influence the tau peptides aggregation, fibrillization and toxicity in vitro. As far as GAGs are concerned, chondroitin sulfate, dermatan sulfate, and heparin seem to influence the peptide aggregation more compared to other GAGs. Although it has been reported that the sulfated form of heparin is found in the amyloid plaques, it did not promote significant aggregation of tau peptides under in vitro conditions. Hyaluronic acid showed the least aggregation promoting effects among the GAGs studied. Further, the GAGs in solution seems to have more influence on the aggregation compared to the surface coated GAGs. Cellular RNA in few $\mathrm{ng} / \mathrm{ml}$ concentrations on the hand, exhibited significant aggregation and toxicity of Tau $\mathrm{V}$ peptides that is known to be a key driver in tau aggregation [5]. Hence, cellular RNA could have potential influence in intracellular tau assembly.

\section{Conclusion}

From this proof of concept study, we show that the polyanions influence the microtuble tau peptide, and mutant peptide aggregation under in vitro conditions. The kind of glycosaminoglycan, and the sulfate modifications, and the physical representation also seems to influence the aggregation mechanism. The studies also show, cellular RNA may to play a significant role in tau aggregation and toxicity. This study could open further opportunities for the investigation of polyanions associated tau aggregation mechanisms and therapeutic interventions. 


\section{Acknowledgement}

We would like to thank the University of Michigan Dearborn department of Mechanical engineering, and the College of Engineering and Computer Science for funding. We would like to thank the University of Michigan Microscope and Imaging (MIL) facility for the use of TEM microscope.

\section{References:}

1. Giacobini, E. \& Gold, G. (2013) Alzheimer disease therapy--moving from amyloidbeta to tau, Nat Rev Neurol. 9, 677-86.

2. Wischik, C. M., Harrington, C. R. \& Storey, J. M. (2014) Tau-aggregation inhibitor therapy for Alzheimer's disease, Biochem Pharmacol. 88, 529-39.

3. Avila, J., Jimenez, J. S., Sayas, C. L., Bolos, M., Zabala, J. C., Rivas, G. \& Hernandez, F. (2016) Tau Structures, Front Aging Neurosci. 8, 262.

4. Iqbal, K., Liu, F., Gong, C. X. \& Grundke-Iqbal, I. (2010) Tau in Alzheimer disease and related tauopathies, Curr Alzheimer Res. 7, 656-64.

5. von Bergen, M., Barghorn, S., Biernat, J., Mandelkow, E. M. \& Mandelkow, E. (2005) Tau aggregation is driven by a transition from random coil to beta sheet structure, Biochim Biophys Acta. 1739, 158-66.

6. Mandelkow, E., von Bergen, M., Biernat, J. \& Mandelkow, E. M. (2007) Structural principles of tau and the paired helical filaments of Alzheimer's disease, Brain Pathol. 17, 83-90.

7. Li, W. \& Lee, V. M. (2006) Characterization of two VQIXXK motifs for tau fibrillization in vitro, Biochemistry. 45, 15692-701.

8. Goux, W. J., Kopplin, L., Nguyen, A. D., Leak, K., Rutkofsky, M., Shanmuganandam, V. D., Sharma, D., Inouye, H. \& Kirschner, D. A. (2004) The formation of straight and twisted filaments from short tau peptides, J Biol Chem. 279, 26868-75.

9. Ganguly, P., Do, T. D., Larini, L., LaPointe, N. E., Sercel, A. J., Shade, M. F., Feinstein, S. C., Bowers, M. T. \& Shea, J. E. (2015) Tau assembly: the dominant role of PHF6 (VQIVYK) in microtubule binding region repeat R3, J Phys Chem B. 119, 458293. 
10. Boutajangout, A. \& Wisniewski, T. (2014) Tau-based therapeutic approaches for Alzheimer's disease - a mini-review, Gerontology. 60, 381-5.

11. Brunden, K. R., Ballatore, C., Crowe, A., Smith, A. B., 3rd, Lee, V. M. \& Trojanowski, J. Q. (2010) Tau-directed drug discovery for Alzheimer's disease and related tauopathies: a focus on tau assembly inhibitors, Exp Neurol. 223, 304-10.

12. Mukrasch, M. D., Biernat, J., von Bergen, M., Griesinger, C., Mandelkow, E. \& Zweckstetter, M. (2005) Sites of tau important for aggregation populate $\{$ beta $\}$-structure and bind to microtubules and polyanions, $J$ Biol Chem. 280, 24978-86.

13. Papy-Garcia, D., Christophe, M., Huynh, M. B., Fernando, S., Ludmilla, S., Sepulveda-Diaz, J. E. \& Raisman-Vozari, R. (2011) Glycosaminoglycans, protein aggregation and neurodegeneration, Curr Protein Pept Sci. 12, 258-68.

14. Kampers, T., Friedhoff, P., Biernat, J., Mandelkow, E. M. \& Mandelkow, E. (1996) RNA stimulates aggregation of microtubule-associated protein tau into Alzheimer-like paired helical filaments, FEBS Lett. 399, 344-9.

15. Ariga, T., Miyatake, T. \& Yu, R. K. (2010) Role of proteoglycans and glycosaminoglycans in the pathogenesis of Alzheimer's disease and related disorders: amyloidogenesis and therapeutic strategies--a review, J Neurosci Res. 88, 2303-15.

16. McLaurin, J., Franklin, T., Zhang, X., Deng, J. \& Fraser, P. E. (1999) Interactions of Alzheimer amyloid-beta peptides with glycosaminoglycans effects on fibril nucleation and growth, Eur J Biochem. 266, 1101-10.

17. Goedert, M., Jakes, R., Spillantini, M. G., Hasegawa, M., Smith, M. J. \& Crowther, R. A. (1996) Assembly of microtubule-associated protein tau into Alzheimer-like filaments induced by sulphated glycosaminoglycans, Nature. 383, 550-3.

18. Paudel, H. K. \& Li, W. (1999) Heparin-induced conformational change in microtubule-associated protein Tau as detected by chemical cross-linking and phosphopeptide mapping, J Biol Chem. 274, 8029-38.

19. Ramachandran, G. \& Udgaonkar, J. B. (2011) Understanding the kinetic roles of the inducer heparin and of rod-like protofibrils during amyloid fibril formation by Tau protein, J Biol Chem. 286, 38948-59.

20. Zhao, J., Huvent, I., Lippens, G., Eliezer, D., Zhang, A., Li, Q., Tessier, P., Linhardt, R. J., Zhang, F. \& Wang, C. (2017) Glycan Determinants of Heparin-Tau Interaction, Biophys J. 112, 921-932.

21. Larini, L., Gessel, M. M., LaPointe, N. E., Do, T. D., Bowers, M. T., Feinstein, S. C. \& Shea, J. E. (2013) Initiation of assembly of tau(273-284) and its DeltaK280 mutant: an experimental and computational study, Phys Chem Chem Phys. 15, 8916-28.

22. Evans, L. D., Wassmer, T., Fraser, G., Smith, J., Perkinton, M., Billinton, A. \& Livesey, F. J. (2018) Extracellular Monomeric and Aggregated Tau Efficiently Enter Human Neurons through Overlapping but Distinct Pathways, Cell Rep. 22, 3612-3624.

23. Avila, J. (2010) Intracellular and extracellular tau, Front Neurosci. 4, 49. 
24. Eschmann, N. A., Do, T. D., LaPointe, N. E., Shea, J. E., Feinstein, S. C., Bowers, M. T. \& Han, S. (2015) Tau Aggregation Propensity Engrained in Its Solution State, $J$ Phys Chem B. 119, 14421-32.

25. Nadimidla, K., Ismail, T. \& Kanapathipillai, M. (2017) Tau peptides and tau mutant protein aggregation inhibition by cationic polyethyleneimine and polyarginine, Biopolymers. 107.

26. Schlachetzki, J. C., Saliba, S. W. \& Oliveira, A. C. (2013) Studying neurodegenerative diseases in culture models, Rev Bras Psiquiatr. 35 Suppl 2, S92-100.

27. Arora, A., Ha, C. \& Park, C. B. (2004) Inhibition of insulin amyloid formation by small stress molecules, FEBS Lett. 564, 121-5.

28. Kizuka, Y., Kitazume, S. \& Taniguchi, N. (2017) N-glycan and Alzheimer's disease, Biochim Biophys Acta.

29. Aguilera, J. J., Zhang, F., Beaudet, J. M., Linhardt, R. J. \& Colon, W. (2014) Divergent effect of glycosaminoglycans on the in vitro aggregation of serum amyloid A, Biochimie. 104, 70-80.

\section{Figure Legends}

Figure 1. A). Tau $V$ peptide (100 $\mu \mathrm{M})$ aggregation kinetics was studied with GAGs (25 $\mu M)$. B). Tau N peptide $(100 \mu M)$ aggregation kinetics was studied with GAGs $(25 \mu M)$. ThS fluorescence, show the aggregation is influenced by the presence of glycosaminoglycan used. Experiments were repeated three times $(n=3)$, and averaged. Here Tau V- VQIVYK; TauN- GKVQIINKLDL; CS-chondroitin sulfate; Hep-heparin; DSDermatan sulfate; HepS- heparan sulfate; HA-hyaluronic acid; ThS-thioflavin S.

Figure 2A. (i). Dynamic light scattering measurements of the Tau N peptide $(100 \mu \mathrm{M})$ aggregates with GAGs $(25 \mu \mathrm{M})$. ii) Tau V peptide $(100 \mu \mathrm{M})$ aggregation with and without GAGs $(25 \mu \mathrm{M})$, assessed by turbidity measurements. The average sizes of each peptide with and without GAGs are tabulated on the right for three experiments. ${ }^{*} p<0.05$. Data 
are expressed as mean \pm SEM.

Figure 3. (i). Turbidity measurements of the Tau $N$ peptide $(100 \mu \mathrm{M})$ aggregates with GAGs $(25 \mu \mathrm{M})$. (ii) Tau V peptide $(100 \mu \mathrm{M})$ aggregation with and without GAGs $(25 \mu \mathrm{M})$, assessed by turbidity measurements. * $p<0.05$. Experiments were performed in triplicates and data are expressed as mean \pm SEM.

Figure 4. TEM images of Tau $\mathrm{N}(100 \mu \mathrm{M})$ peptide aggregation in the presence of GAGs $(25 \mu \mathrm{M})$. The aggregates were made in MOPS buffer at $37^{\circ} \mathrm{C}$, and the samples were spotted on holy carbon grids, and negatively stained with $1 \%$ uranyl formate prior to imaging. Scale bar $1 \mu \mathrm{m}$.

Figure 5. TEM images of Tau $V(100 \mu \mathrm{M})$ peptide aggregation in the presence of GAGs $(25 \mu \mathrm{M})$. The samples were aggregated in MOPS buffer at $37^{\circ} \mathrm{C}$, and TEM images were obtained to assess the morphology using the similar procedure used for Tau N samples. Scale bar $1 \mu \mathrm{m}$.

Figure 6. Toxicity effect of Tau peptides aggregates with GAGs on SH-SY5Y cells. A) Tau N with GAGs B) Tau $\vee$ with GAGs. * $p<0.05$. Data are expressed as mean $\pm \mathrm{SEM}$, for three experiments.

This article is protected by copyright. All rights reserved. 


\section{$\operatorname{Kapp}\left(\min ^{-1}\right)$}

Figure 7. (A). Tau $\mathrm{N}$ peptide $(100 \mu \mathrm{M})$ aggregation kinetics was studied with RNA concentrations of $0.25 \mathrm{ng} / \mathrm{ml}$ and $0.5 \mathrm{ng} / \mathrm{ml}$. (B). Tau $\mathrm{V}$ peptide $(100 \mu \mathrm{M})$ aggregation kinetics was studied with RNA concentrations of $0.25 \mathrm{ng} / \mathrm{ml}$ and $0.5 \mathrm{ng} / \mathrm{ml}$. ThS fluorescence, reveal the Tau $\mathrm{V}$ aggregation is significantly influenced by the presence of RNA. (C). AFM images of Tau $\mathrm{N}$ and Tau $\mathrm{V}$ peptide aggregation in the presence of RNA.

Figure 8. Toxicity effect of Tau peptides aggregates in the presence of RNA on SHSY5Y cells. Tau $\mathrm{N}$ and Tau $\mathrm{V}$ at $16 \mu \mathrm{M}$ concentrations were incubated with and without $0.8 \mathrm{ng} / \mathrm{ml}$ RNA, and toxicity was performed after 48 hours. ${ }^{*} \mathrm{p}<0.05$ indicate significance, determined from three independent experiments, and data are expressed as mean \pm SEM.

Table 1: Apparent rate constant of tau peptides aggregation with GAGs and RNA

Note: Here Tau V-100 $\mu \mathrm{M}$; CS, Hep - $25 \mu \mathrm{M}$; RNA-0.5 ng/ml. Tau V and Tau N alone exhibited negligible Kapp values, indicting very slow kinetics in the absence of GAGs or RNA, and hence not reported in the table.

This article is protected by copyright. All rights reserved. 


\begin{tabular}{ccc|ccc}
\hline TauV: CS & TauV: Hep & TauV: RNA & TauN: CS & TauN: Hep & TauN:RNA \\
1 & 0.45 & 0.625 & 0.06 & 0.15 & 0.15 \\
\hline
\end{tabular}

This article is protected by copyright. All rights reserved. 
A. Tau V-VQIVYK

$\rightarrow-$ Tau V + Cs

$--x-$ Tau V + Hep

... - Tau V+ DS

$-\square-$ Tau V + Heps

$\odot$ Tau V + HA

- $\_$TauV

$\rightarrow-$ ThS

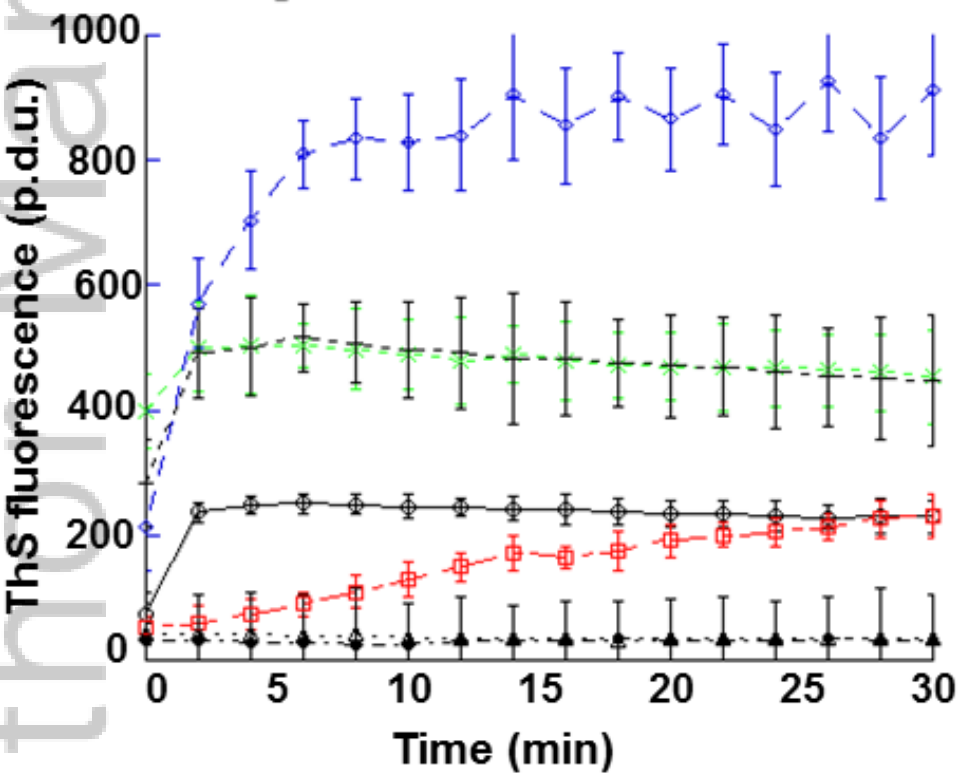

B. Tau N-GKVQIINKLDL
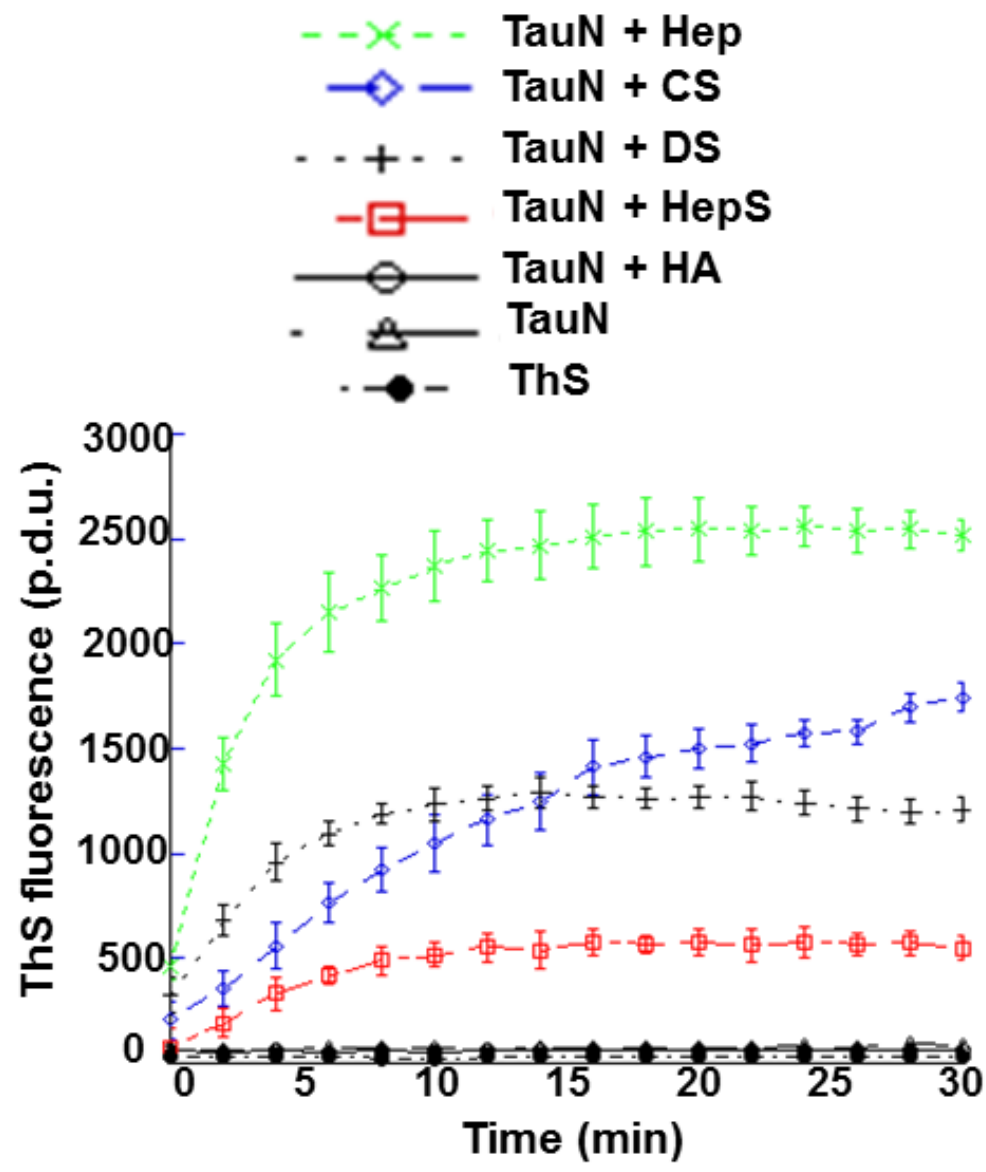


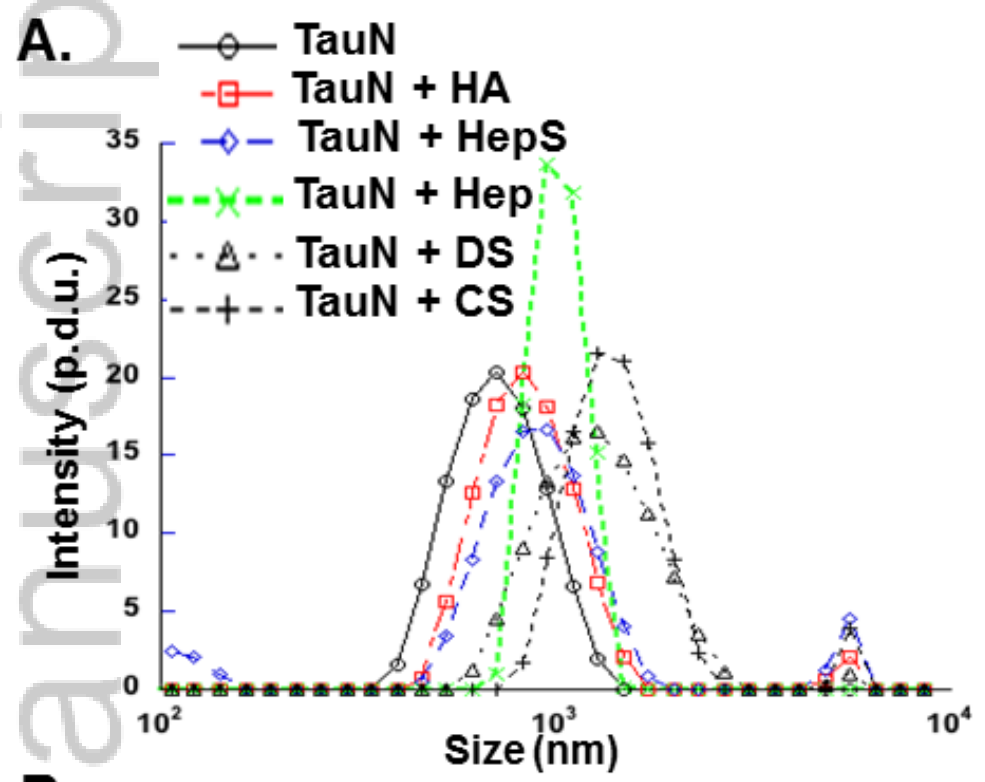

\begin{tabular}{|l|l|}
\hline Peptide/GAGS & Size $(\mathbf{n m})$ \\
\hline TauN & $903 \pm 69$ \\
\hline TauN + HA & $1128 \pm 134$ \\
\hline TauN + HepS & $1081 \pm 207$ \\
\hline TauN + Hep & $1291 \pm 171$ \\
\hline TauN + DS & $1300 \pm 50$ \\
\hline TauN + CS* & $1789 \pm 81$ \\
\hline
\end{tabular}

B.

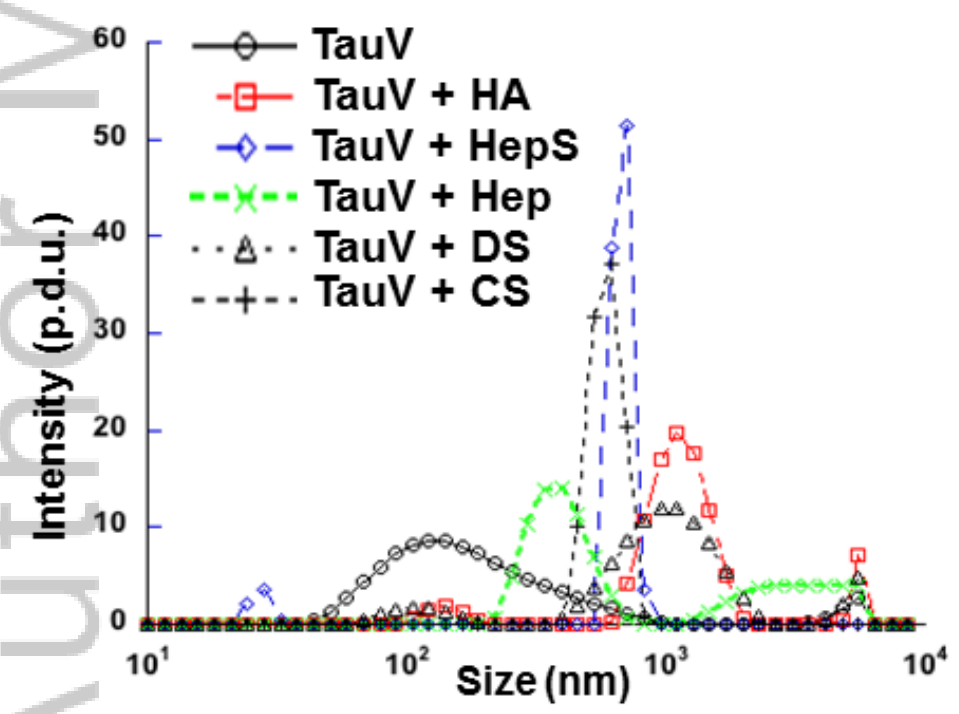

\begin{tabular}{|l|l|}
\hline Peptide/GAGS & Size $(\mathbf{n m})$ \\
\hline TauV & $700 \pm 250$ \\
\hline TauV + HA & $1846 \pm 210$ \\
\hline TauV + HepS & $1950 \pm 360$ \\
\hline TauV + Hep & $1450 \pm 130$ \\
\hline TauV + DS & $1765 \pm 150$ \\
\hline TauV + CS * & $2100 \pm 160$ \\
\hline
\end{tabular}



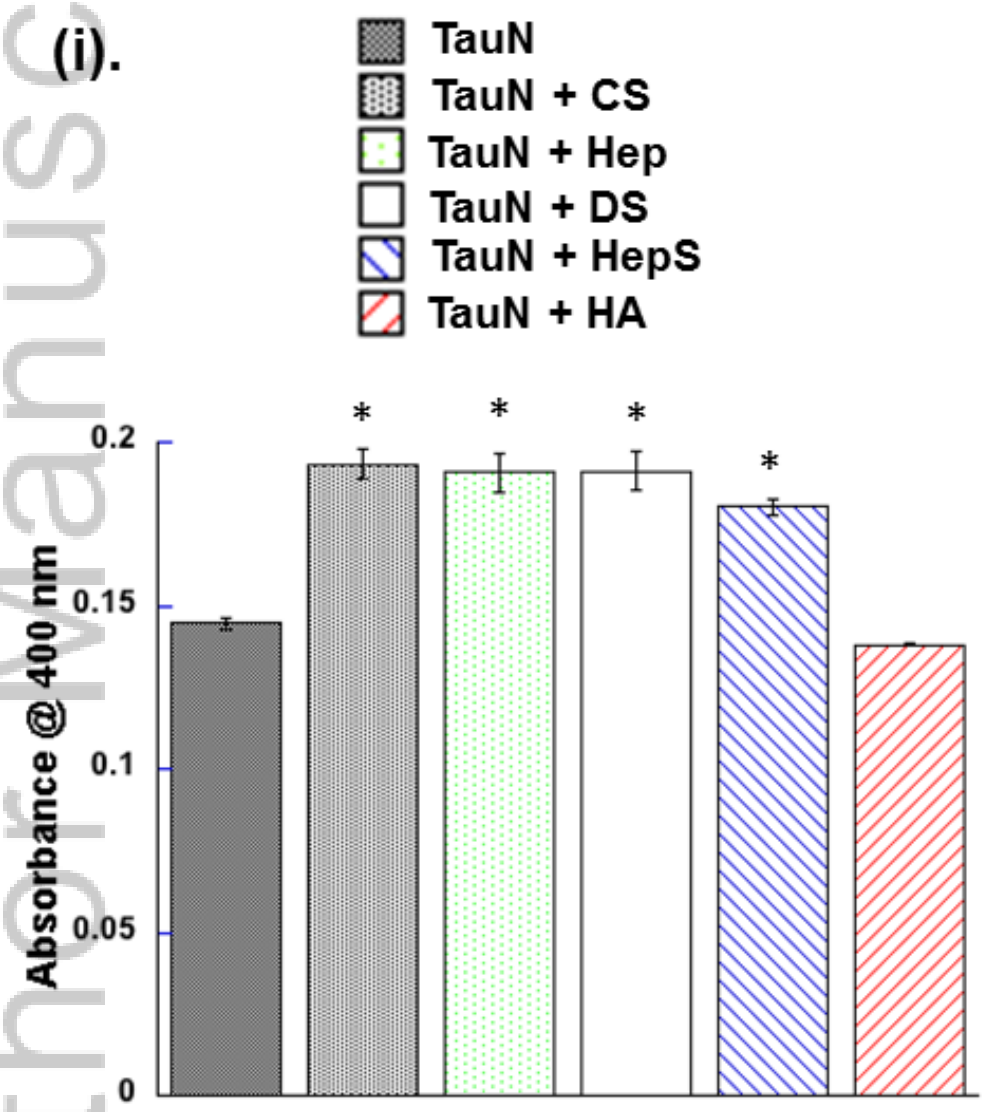
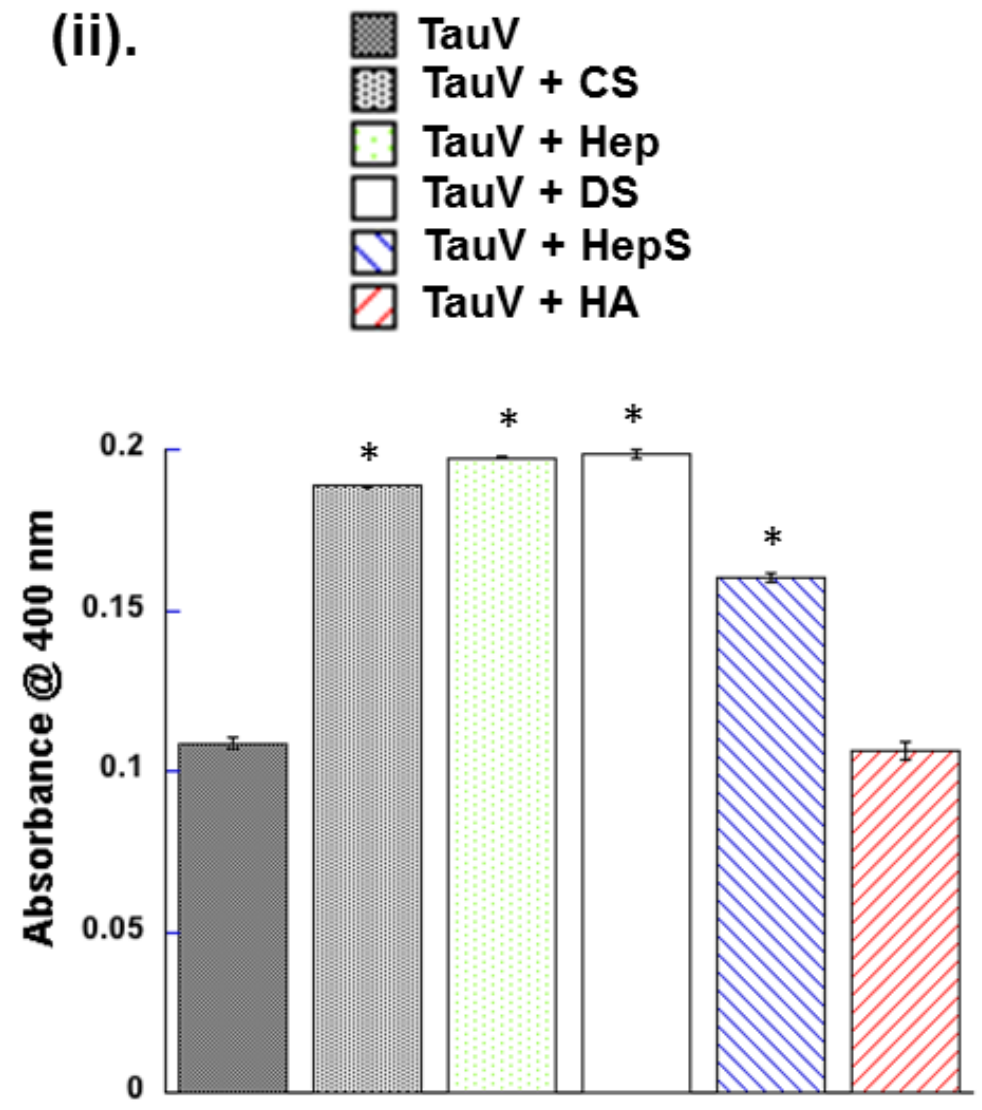

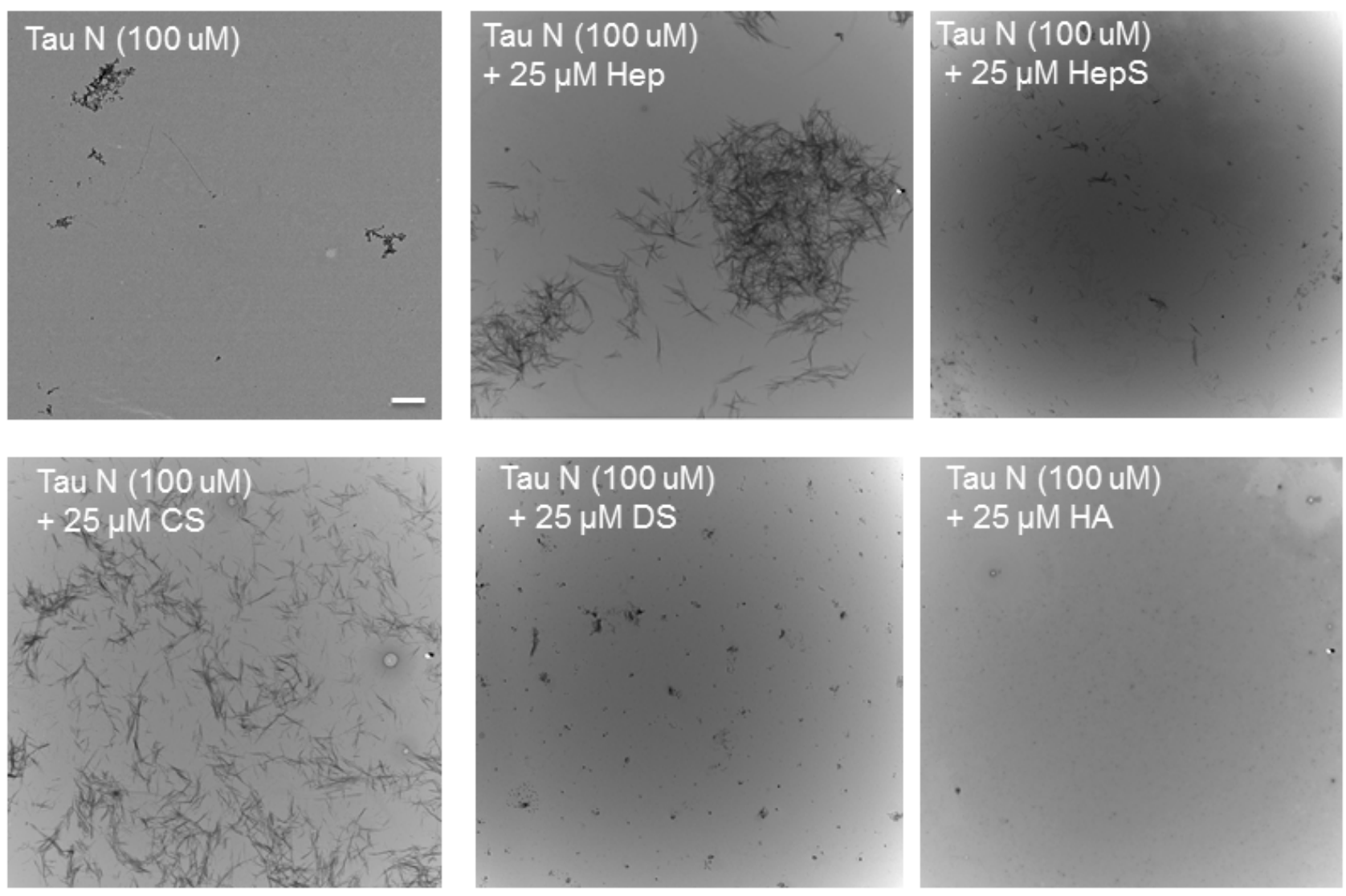

This article is protected by copyright. All rights reserved. 

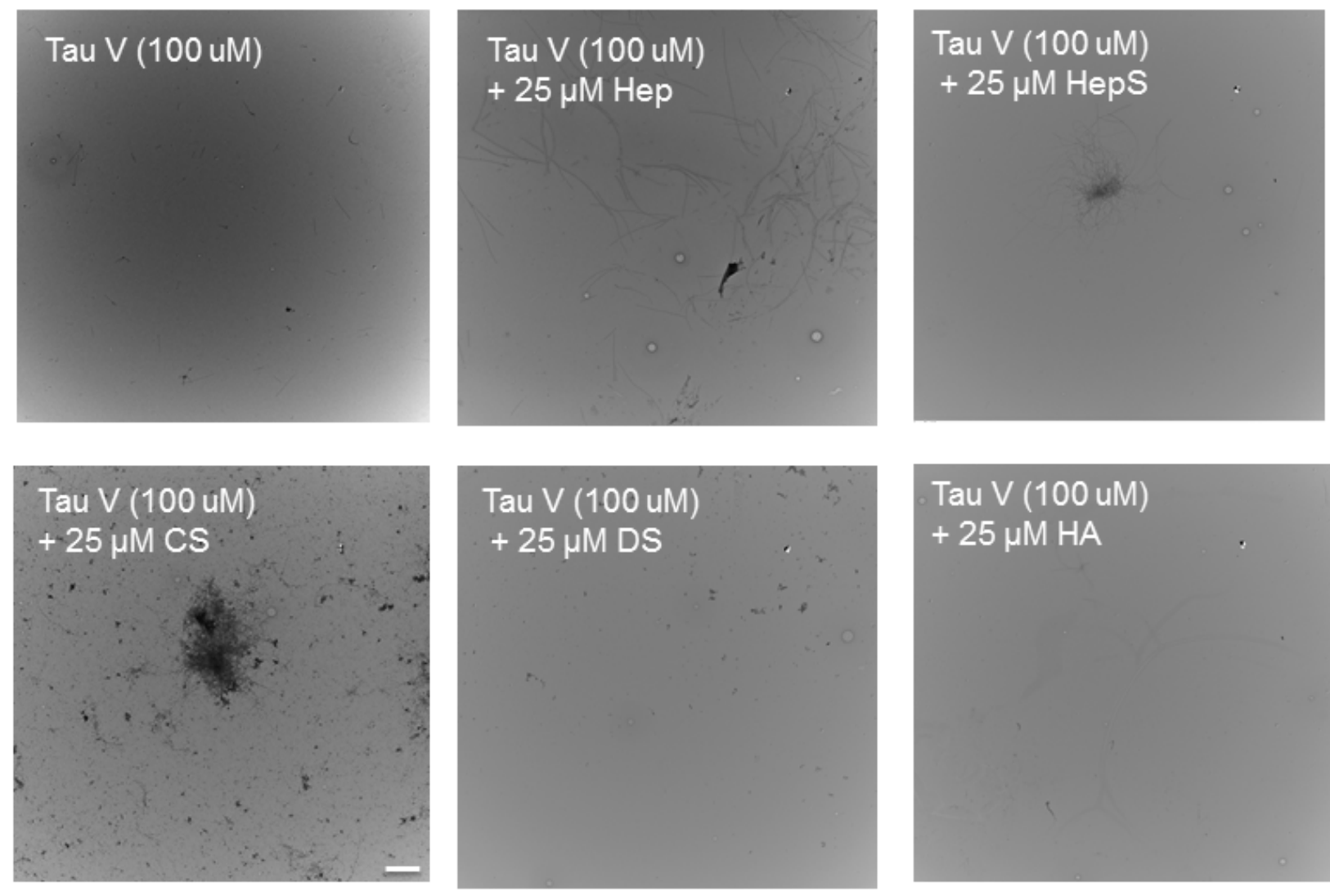

This article is protected by copyright. All rights reserved. 
A.

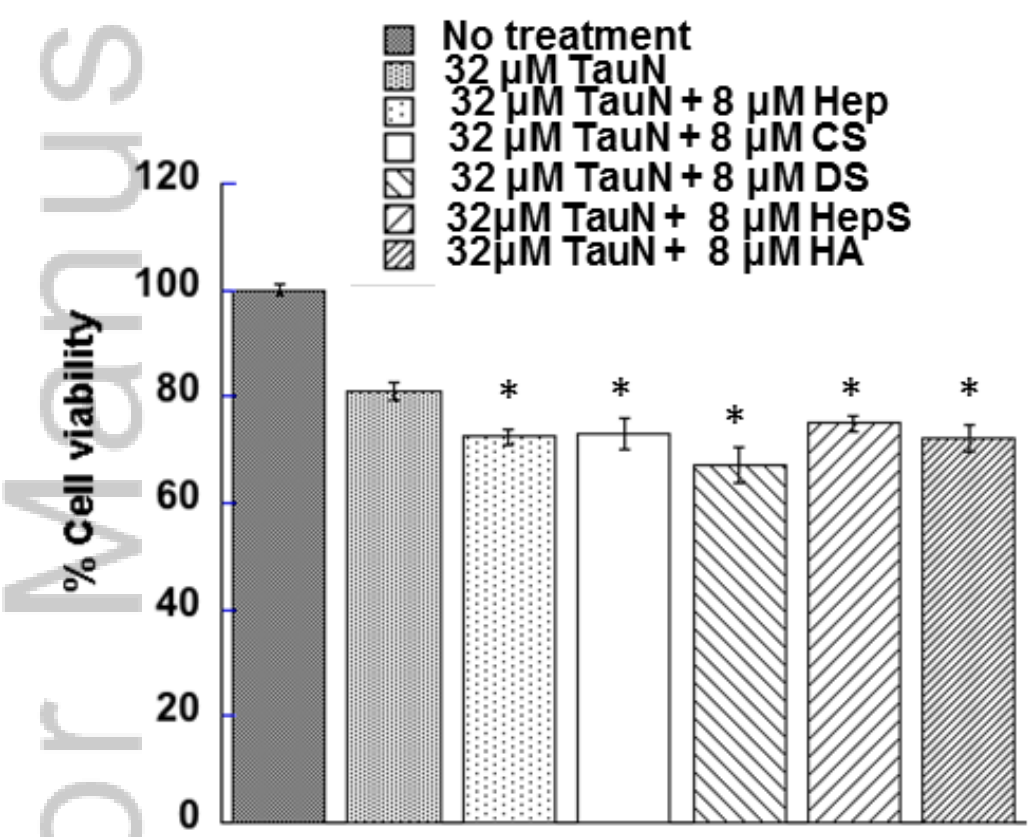

B.

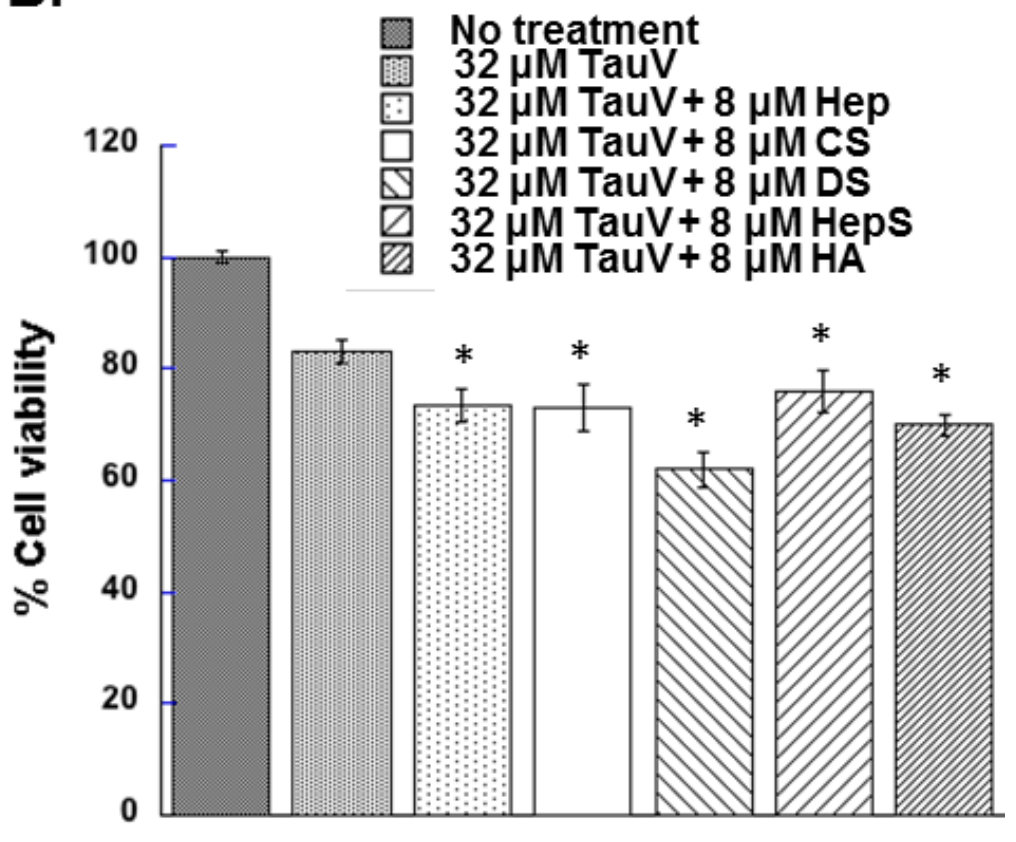




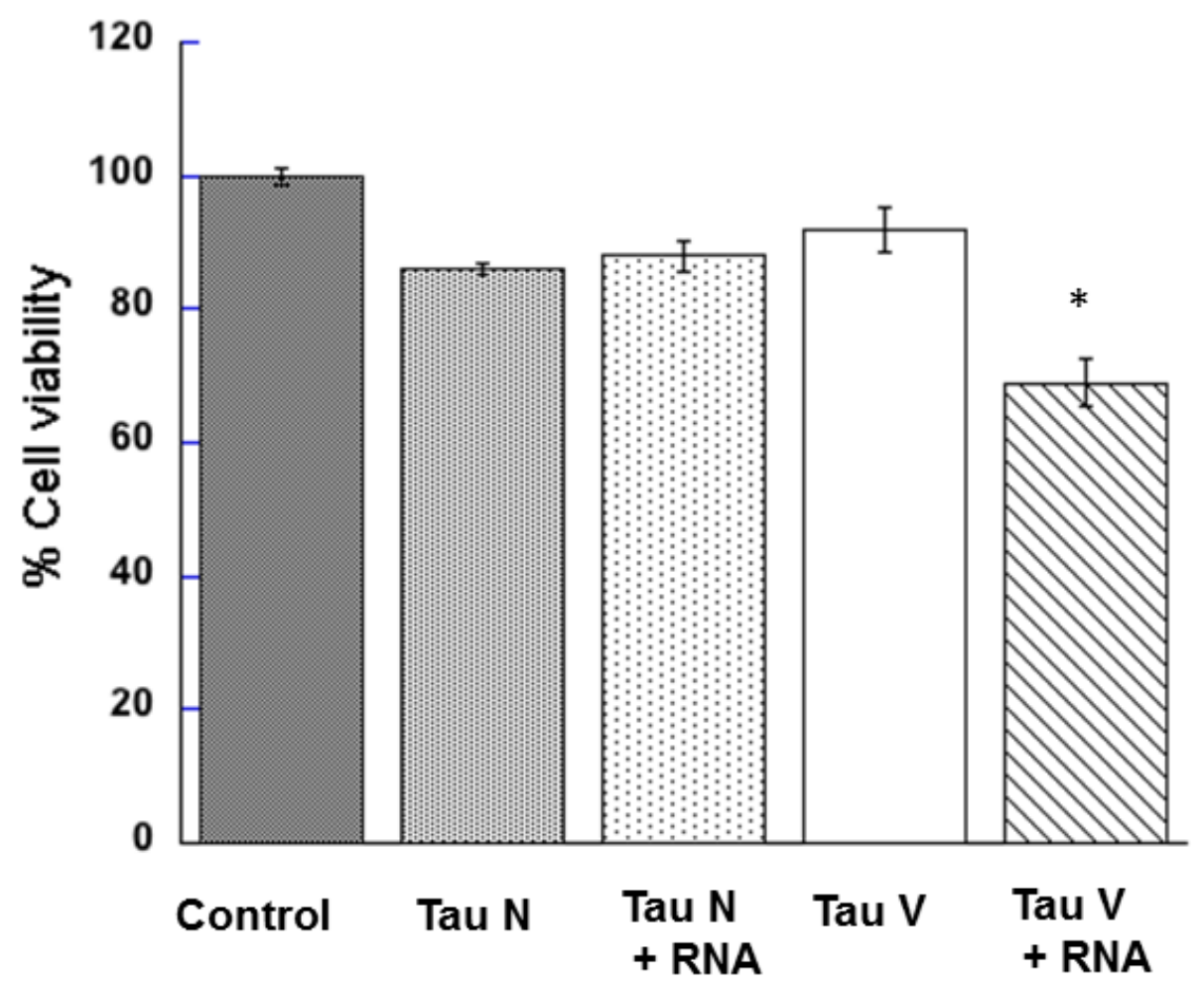

This article is protected by copyright. All rights reserved. 


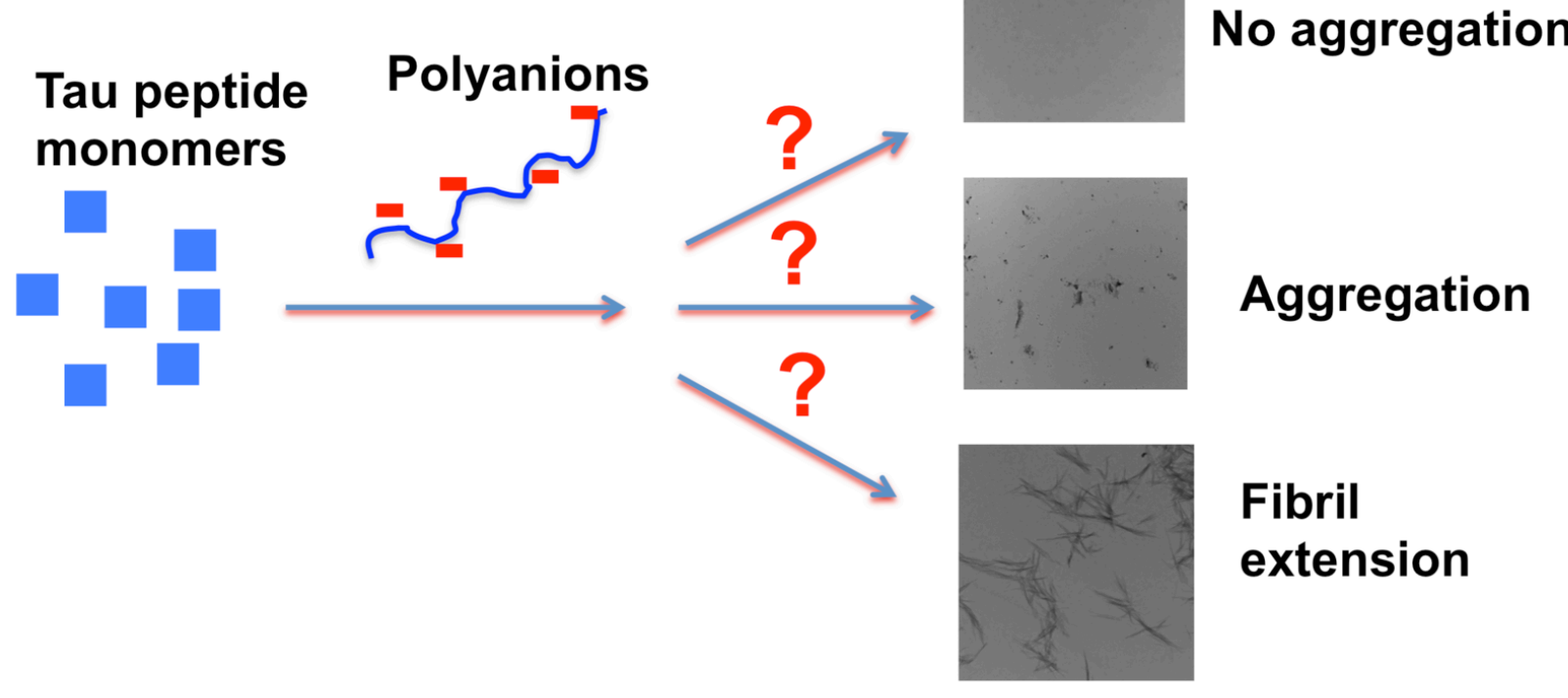

PSC_3125_GTOC.tiff

This article is protected by copyright. All rights reserved. 